\title{
Memory for individual items is related to nonreinforced preference change
}

\author{
Rotem Botvinik-Nezer, ${ }^{1,2,3}$ Akram Bakkour, ${ }^{4,5}$ Tom Salomon, ${ }^{2}$ Daphna Shohamy, ${ }^{4,6,7}$ \\ and Tom Schonberg ${ }^{1,2}$
}

${ }^{1}$ Sagol School of Neuroscience, Tel Aviv University, Tel Aviv 6997801, Israel; ${ }^{2}$ School of Neurobiology, Biochemistry, and Biophysics, Faculty of Life Science, Tel Aviv University, Tel Aviv 6997801, Israel; ${ }^{3}$ Department of Psychological and Brain Sciences, Dartmouth College, Hanover, New Hampshire 03755, USA; ${ }^{4}$ Department of Psychology, Columbia University, New York, New York 10027, USA; ${ }^{5}$ Department of Psychology, the University of Chicago, Chicago, Illinois 60637, USA; ${ }^{6}$ Mortimer B. Zuckerman Mind Brain Behavior Institute; ${ }^{7}$ the Kavli Institute for Brain Science, Columbia University, New York, New York 10027, USA

\begin{abstract}
It is commonly assumed that memories contribute to value-based decisions. Nevertheless, most theories of value-based decision-making do not account for memory influences on choice. Recently, new interest has emerged in the interactions between these two fundamental processes, mainly using reinforcement-based paradigms. Here, we aimed to study the role memory processes play in preference change following the nonreinforced cue-approach training (CAT) paradigm. In CAT, the mere association of cued items with a speeded motor response influences choices. Previous studies with this paradigm showed that a single training session induces a long-lasting effect of enhanced preferences for high-value trained stimuli, that is maintained for several months. We hypothesized that CAT increases memory of trained items, leading to enhanced accessibility of their positive associative memories and in turn to preference changes. In two preregistered experiments, we found evidence that memory is enhanced for trained items and that better memory is correlated with enhanced preferences at the individual item level, both immediately and 1 mo following CAT. Our findings suggest that memory plays a central role in value-based decision-making following CAT, even in the absence of external reinforcements. These findings contribute to new theories relating memory and value-based decision-making and set the groundwork for the implementation of novel nonreinforced behavioral interventions that lead to long-lasting behavioral change.
\end{abstract}

[Supplemental material is available for this article.]

Value-based decision-making and memory are both extensively studied processes in cognitive psychology and cognitive neuroscience (Fellows 2017). Most theories of value-based decision-making have focused on processes related to the incremental learning of value following external reinforcement, but have not explicitly addressed the role of memory per se. Thus, fundamental questions remain regarding interactions between memory and value-based decisions, which have been gaining attention in recent years.

Several recent empirical studies have demonstrated interactions between episodic memory and value-based decision-making. For example, memory for past events has been shown to bias valuebased decisions (Duncan and Shohamy 2016), differently for choices of novel versus choices of familiar options (Duncan et al. 2019), and choice behavior and fMRI signals during value-based decision-making were better explained by episodic memory for individual past choices than by a standard reinforcement learning model (Bornstein et al. 2017). Another study has found that during sampling of episodic memories of previous choices, the retrieved context influenced present choices, deviating from the predictions of standard reinforcement learning models (Bornstein and Norman 2017). Other studies have demonstrated that the long time known effect of choices on future preferences is related to memory processes (Chammat et al. 2017; DuBrow et al. 2019; Luettgau et al. 2020). At the neural level, the ventromedial prefron-

Corresponding author: schonberg@tauex.tau.ac.il

Article is online at http://www.learnmem.org/cgi/doi/10.1101/lm.053411.121. Freely available online through the Learning \& Memory Open Access option. tal cortex (vmPFC) and the hippocampus both have been shown to play a role in memory processes and value-based decisions (Weilbächer and Gluth 2017) and recent studies have been further emphasizing that the hippocampus bridges between past experience and future decisions (Bakkour et al. 2019; Biderman et al. 2020).

All these studies, and many others, highlighted the interaction between memory and value-based decision-making involving external reinforcements. However, everyday life involves decisions and associations that are not directly reinforced. Thus, it remains unclear whether memory plays a general role in value-based decision-making even without external reinforcements.

To better understand the role of memory processes in shaping preferences independently of external reinforcements, we used a novel behavioral change paradigm, named cue-approach training (CAT). In this paradigm, associating images of items with a neutral cue and a speeded motor response results in a consistent preference enhancement without external reinforcement, which is maintained for months (Schonberg et al. 2014; Bakkour et al. 2018; Salomon et al. 2018, 2019; Botvinik-Nezer et al. 2020). During CAT, images of items are consistently paired with a neutral cue and a speeded motor response ("Go items"), while other items are presented without the cue or the response ("NoGo items").

(C) 2021 Botvinik-Nezer et al. This article, published in Learning \& Memory, is available under a Creative Commons License (Attribution-NonCommercial 4.0 International), as described at http://creativecommons.org/licenses/by$\mathrm{nc} / 4.0 /$. 
One training session with several presentations of all items leads to long-lasting preference changes, measured as the likelihood of choosing Go over NoGo items that had similar initial subjective values (Schonberg et al. 2014). Results from over 30 samples with this paradigm have demonstrated a replicable effect on various types of stimuli, including snack food items, fruits and vegetables, unfamiliar faces, fractal art images, and positive affective images (Bakkour et al. 2016, 2017; Veling et al. 2017; Zoltak et al. 2017; Bakkour et al. 2018; Salomon et al. 2018, 2019; Botvinik-Nezer et al. 2020), revealing the potential of the CAT paradigm as an experimental platform for value-based decision-making without external reinforcements (Schonberg and Katz 2020).

The underlying mechanisms of the change of preferences following CAT are not yet fully understood (Schonberg et al. 2014; Bakkour et al. 2017; Salomon et al. 2019; Botvinik-Nezer et al. 2020; Schonberg and Katz 2020). The long-lasting nature of the effect, which has been shown to last for up to 6 mo following a single training session (Schonberg et al. 2014; Salomon et al. 2018, 2019; Botvinik-Nezer et al. 2020), raises the hypothesis that memory processes are involved in its maintenance. Furthermore, previous studies have found enhanced memory for Go compared with NoGo items with other types of Go-NoGo tasks (Chiu and Egner 2015a,b; Yebra et al. 2019) and for items for which participants have a sense of agency (Murty et al. 2015). One recent study provided preliminary evidence suggesting that memory is involved in preference change following a similar nonreinforced Go/NoGo training task (Chen et al. 2021).

We hypothesized that CAT enhances memory of Go items, which in turn leads to preferring these items over NoGo items. Previous neuroimaging findings with CAT that suggested possible interactions between hippocampal fMRI activity and subsequent preferences 1 mo following CAT, provide additional evidence in support of this hypothesis (Botvinik-Nezer et al. 2020). Therefore, here we set out to test the role memory processes play in the behavioral change of preferences following CAT, in the short and in the long term.

We propose an underlying mechanism for the CAT effect, in which preference change following CAT results from a boost in memory encoding of positive Go items, which in itself is a consequence of enhanced perceptual processing of Go items (Schonberg et al. 2014; Botvinik-Nezer et al. 2020). We hypothesize that the enhanced encoding of Go items, as well as the greater perceptual activation in response to them, increases accessibility of attributes and associations of these specific Go items (Anderson 1983; Bhatia 2013). Furthermore, we hypothesized that preference changes, reflected in the binary choice phase, are due to the enhanced accessibility of memory associations of the Go items, which tips the scales in favor of the Go items when the associations are positive.

In order to test memory for individual items, in the current work we introduced a memory recognition task following CAT. In two independent preregistered experiments and one pilot experiment, memory was evaluated following a long (16 repetitions) or short (a single exposure) CAT training session, before the probe phase that evaluated post-training preferences. We then tested our predictions that (1) memory will be stronger for Go compared with NoGo items following CAT (more accurate and faster responses in the recognition task) and (2) that memory will be related to choices (better remembered Go items will be chosen over worse remembered NoGo items). Since the link between better memory and enhanced choices is hypothesized to be related to positive associated memories, we tested the relationship between memory and choices separately for choices between low-value and choices between high-value items. These hypotheses were tested both in the short term (immediately or a few days after CAT) and in a 1-mo follow-up.

\section{Results}

\section{Experiment 1}

In the first preregistered experiment, we tested whether memory was enhanced for Go compared with NoGo items following CAT and whether memory was related to choices. This experiment included three sessions: Session 2 was completed exactly $3 \mathrm{~d}$ after session 1 and tested memory involvement in the (relatively) short-term effect of CAT. The follow-up session, testing the longterm effects, was performed $\sim 1$ mo following session 1 .

Thirty-nine participants completed the first two sessions. Four participants were excluded based on preregistered exclusion criteria. Thirty participants also completed the follow-up session, $\sim 1$ mo after session $1(M=34.3 \mathrm{~d}, \mathrm{SD}=5.04 \mathrm{~d}$, range $=28-46 \mathrm{~d})$ (demographic statistics are reported in Table 1$)$.

In session 1, we first evaluated participants' preferences for 60 snack food items with a Becker-DeGroot-Marschak (BDM) auction procedure (Fig. 1A; Becker et al. 1964). Then, participants completed 16 repetitions of CAT with 40 of the 60 snack food items (Fig. 1B). In session 2 , we tested participants' memory with a recognition task (Fig. 1C) that included 28 new and 28 old (i.e., previously presented) snack food items. We then tested whether their preferences were modified with a binary forced choice probe task (Fig. 1D; see Supplemental Table 1 for item allocation to value categories), and finally an additional BDM auction (Fig. 1A). The follow-up session included the same tasks as in session 2 .

\section{Recognition}

Our recognition analyses focused on the binarized version of participants' responses in the old/new recognition task (for details about the analysis, see "Recognition Analysis" in the Materials and Methods; for results of the Go/NoGo recognition task, see "Supplemental Exploratory Analyses" in the Supplemental Material).

\section{Session 2 (for full statistics, see Supplemental Table 2)}

Overall performance. The mean hit rate of participants was $86.31 \%$ $(\mathrm{SD}=10.33 \%)$. The mean correct rejection rate was $70.53 \%(\mathrm{SD}=$ $17.60 \%)$. The mean $\mathrm{d}^{\prime}$ was $1.824(\mathrm{SD}=0.747)$. The mean $\mathrm{RT}$ was $1.458 \mathrm{sec}(\mathrm{SD}=0.262 \mathrm{sec})$ for hits, $1.796 \mathrm{sec}(\mathrm{SD}=0.504 \mathrm{sec})$ for misses, 1.589 ( $\mathrm{SD}=0.351) \mathrm{sec}$ for correct rejections and $1.840 \mathrm{sec}$ $(\mathrm{SD}=0.366 \mathrm{sec})$ for false alarms.

Hit rate. Overall, as predicted and preregistered, hit rate in the old/new recognition task was significantly higher for Go $(M=88.51 \%)$ compared with NoGo $(M=84.97 \%)$ items (one-sided $P=0.008$, odds ratio $=2.873$, 95\% CI [1.225, 6.737], mixed-effects logistic regression) (see Fig. 2A), while it was not higher for high-value $(\mathrm{M}=86.30 \%)$ compared with low-value $(M=87.40 \%)$ items $(P=0.437)$. No interaction was found between the value category (high-value/low-value) and item type (Go/NoGo; two-sided $P=0.472$ ).

Response times. In accordance with our preregistered predictions, RTs of correct responses in the recognition task were significantly faster for Go $(M=1.392 \mathrm{sec})$ compared with NoGo $(M=1.514 \mathrm{sec})$ items (one-sided $P=0.001$, estimated mean difference $=-0.124$, mixed-effects linear regression) (see Fig. 2B). We did not find RT differences between high-value $(M=1.448 \mathrm{sec})$ and low-value $(M=1.454 \mathrm{sec})$ items (one-sided $P=0.435$, estimated mean difference $=-0.005)$ or an interaction between the value category and item type (two-sided $P=0.577$ ). 
Table 1. Demographic information

\begin{tabular}{lccccc}
\hline & & & & \multicolumn{2}{c}{ Follow-up } \\
\cline { 3 - 5 } & Sample size (excluded) & Females (percent) & Age $\boldsymbol{M}(\mathrm{SD})$ & Sample size & Interval in days $\boldsymbol{M}$ (range) \\
\hline Pilot experiment & $25(2)$ & $14(56 \%)$ & $25.52(3.24)$ & 14 & $45.7(27-68)$ \\
Experiment 1 & $35(4)$ & $26(74.3 \%)$ & $24.63(5.26)$ & 30 & $34.3(28-46)$ \\
Experiment 2 & $35(3)$ & $25(71.4 \%)$ & $24.69(4.61)$ & - & - \\
\hline
\end{tabular}

Follow-up session (for full statistics, see Supplemental Table 2)

In this session, we tested the relationships between memory and choices in the long term, 1 mo following CAT.

Overall performance. The mean hit rate of participants was $84.47 \%$ $(\mathrm{SD}=11.76 \%)$. The mean correct rejection rate was $61.15 \%$

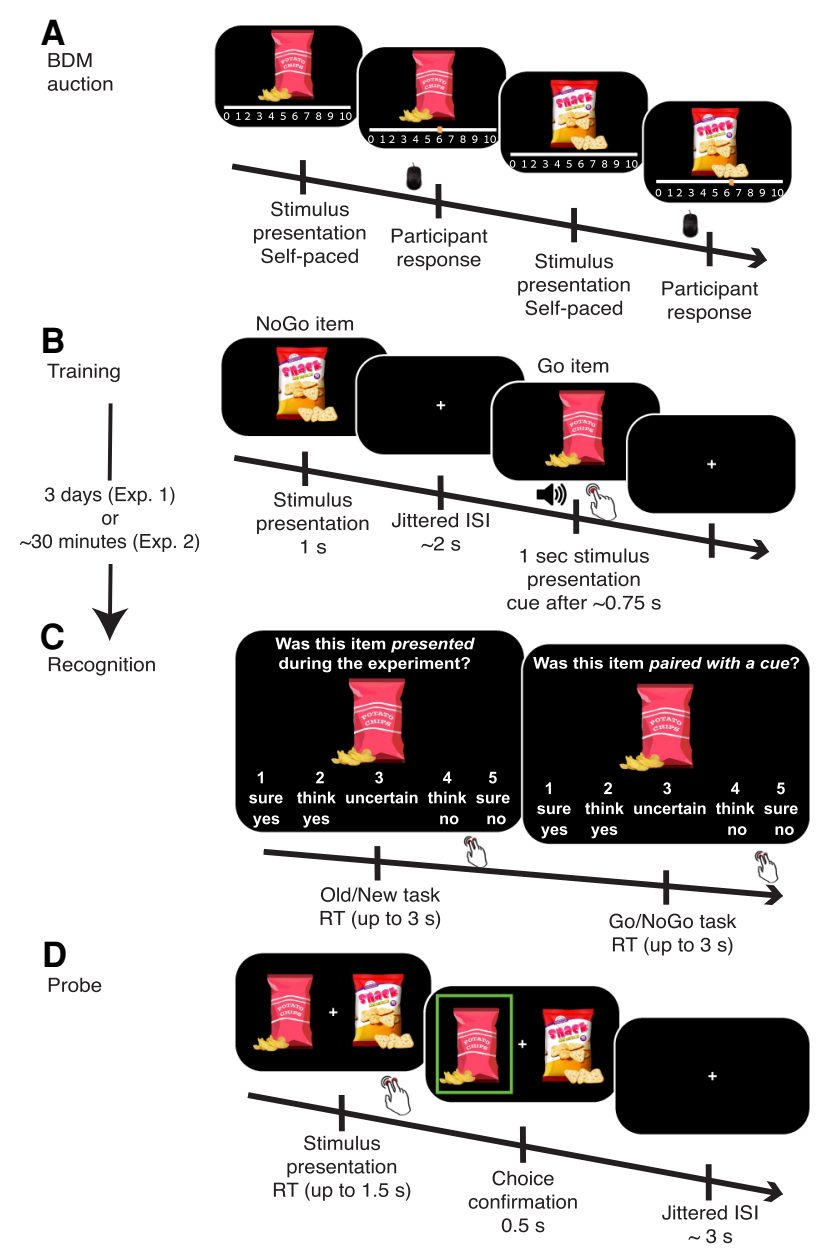

Figure 1. Outline of the experimental procedures. $(A)$ Initial preferences were evaluated using the Becker-Degroot-Marschak (BDM) auction procedure. (B) Cue approach training (CAT): Go items were associated with a neutral auditory cue and a speeded response (16 repetitions per stimulus in experiment 1 , and only one repetition per stimulus in experiment 2 ). (C) Recognition memory task. (D) Probe: Participants chose between pairs of Go and NoGo items of similar initial value. In experiment 1, the recognition and probe tasks were completed in session 2, $3 \mathrm{~d}$ following the first session, and then repeated again in the follow-up session, $\sim 1$ mo following the first session. In experiment 2, all tasks were performed on the same day, and a 30-min filler task separated between the training and the recognition task.
$(\mathrm{SD}=19.85 \%)$. The mean $d^{\prime}$ was $1.454(\mathrm{SD}=0.742)$. The mean RT was $1.456 \mathrm{sec}(\mathrm{SD}=0.314 \mathrm{sec})$ for hits, $1.633 \mathrm{sec}(\mathrm{SD}=0.446 \mathrm{sec})$ for misses, $1.603 \mathrm{sec}(\mathrm{SD}=0.326 \mathrm{sec})$ for correct rejections and $1.749 \mathrm{sec}(\mathrm{SD}=0.336 \mathrm{sec})$ for false alarms.

Hit rate. As in session 2 and in accordance with our preregistered predictions, hit rate in the old/new recognition task was significantly higher for Go $(M=88.81 \%)$ compared with NoGo $(M=83.53 \%)$ items (one-sided $P=0.017$, odds ratio $=1.981,95 \%$ CI $[1.055,3.718]$, mixed-effects logistic regression) (see Fig. 2C) and not significantly higher for high-value $(M=87.57 \%)$ compared with low-value $(M=84.77 \%)$ items (one-sided $P=$ 0.123 , odds ratio $=1.379,95 \% \mathrm{CI}[0.788,2.412])$. As in session 2 , there was no interaction between the value category and item type (two-sided $P=0.394$ ).

Response times. A general trend of faster RTs for correct responses for Go $(M=1.422 \mathrm{sec})$ comparedwith NoGo $(\mathrm{M}=1.477 \mathrm{sec})$ items was observed, but only with marginal significance (one-sided $P=$ 0.063 , estimated mean difference $=-0.049$, mixed-effects linear regression) (see Fig. 2D). RTs were not significantly faster for high-value $(\mathrm{M}=1.442 \mathrm{sec})$ compared with low-value $(M=1.455)$ items (one-sided $P=0.314$, estimated mean difference $=-0.015$ ). There was no interaction between the value category and item type $(P=0.636)$.

Choices—all sessions (see Fig. 3; for full statistics see Supplemental Table 3) As predicted and preregistered, replicating previous findings with CAT (Schonberg et al. 2014; Salomon et al. 2018; Botvinik-Nezer et al. 2020), participants significantly chose high-value Go over high-value NoGo items, both $3 \mathrm{~d}$ after CAT $(M=61.67 \%$, one-sided $P<0.001$, odds ratio $=1.745,95 \%$ CI $[1.307,2.331]$, mixed-effects logistic regression) and 1 mo after CAT $(M=59.62 \%$, one-sided $P<0.001$, odds ratio $=1.546,95 \%$ CI $[1.208,1.978])$. They also significantly chose low-value Go over low-value NoGo items $3 \mathrm{~d}$ after CAT $(M=58.16 \%$, one-sided $P=0.009$, odds ratio $=1.487,95 \%$ CI $[1.071,2.065])$ and marginally in the 1 -mo follow-up $(M=$ $54.95 \%$, one-sided $P=0.066$, odds ratio $=1.244,95 \%$ CI $[0.936$, 1.654]). Unlike previous experiments with snack food items, the proportion of Go item choices was not significantly higher for high-value compared with low-value probe pairs in either one of the two sessions. However, it should be noted that we improved the models used in previous studies, which is likely the reason for the lack of replication.

\section{Relationships between memory and choices}

Our main analysis focused on the relationships between memory and choices at the individual item level.

\section{Session 2 (see Supplemental Table 4)}

We tested the relationship between memory for the specific items and choices made in the subsequent probe in session 2 . Based on our suggested underlying mechanism of CAT, we predicted that 
Experiment 1 - Recognition Results
A

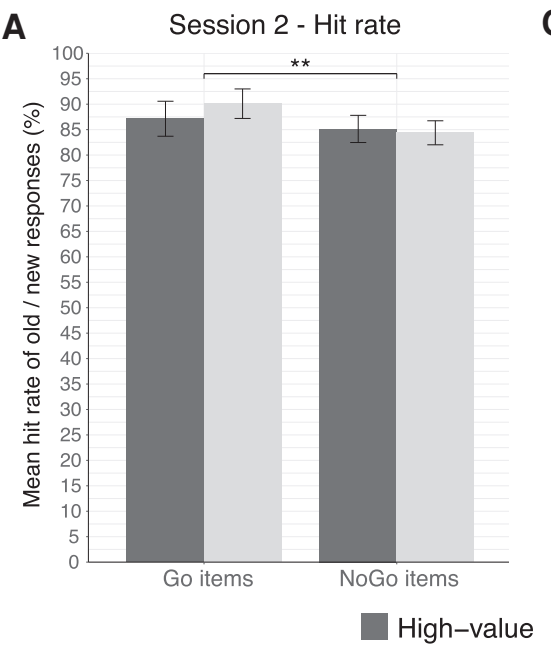

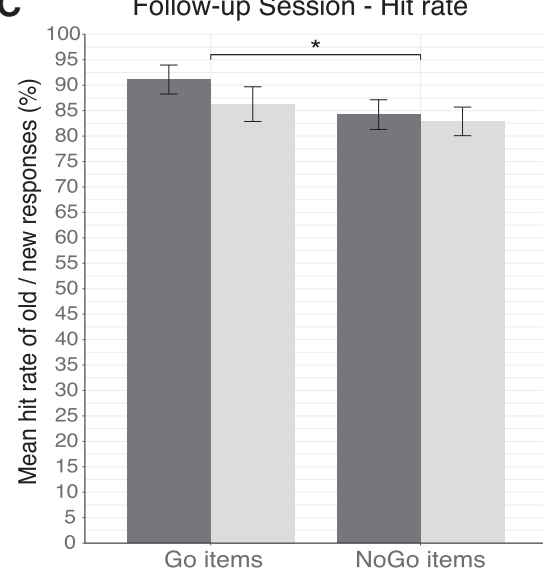

Low-value
C Follow-up Session - Hit rate
B

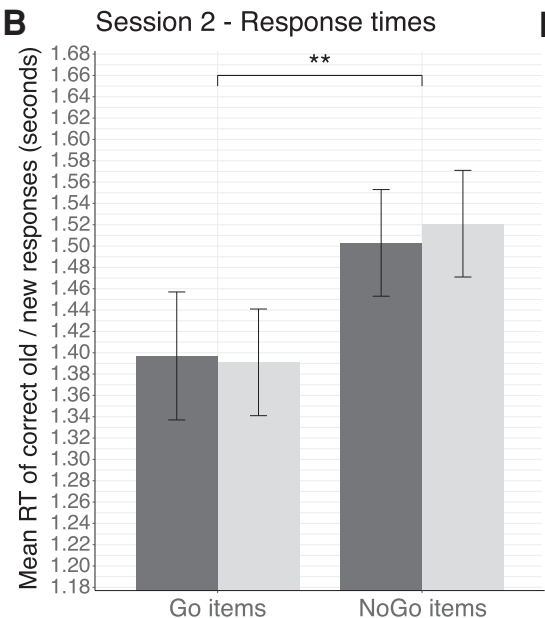

D Follow-up Session - Response times

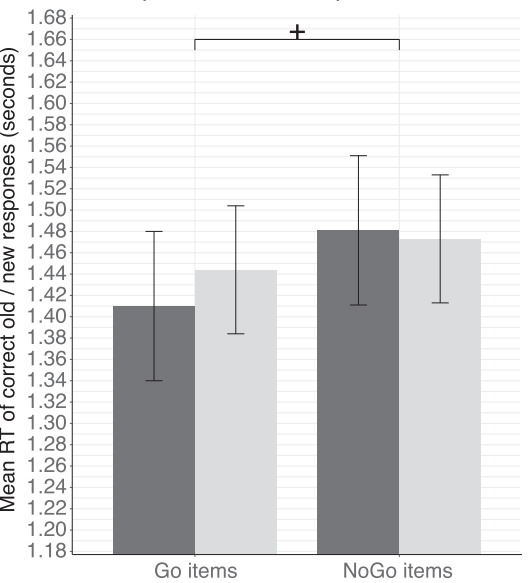

Figure 2. Recognition results of experiment 1 . The mean hit rate $(A, C)$ and response times $(B, D)$ of each participant were calculated and then averaged across participants. Error bars represent standard error of the mean across participants. Asterisks represent one-sided statistical significance. $(+) P<0.07$, $\left(^{*}\right) P<0.05,\left({ }^{*}\right) P<0.01$. these relationships will be significantly positive for choices between high-value items. We further predicted a significant interaction between memory and the pair value category (high-value/ low-value).

Participants chose Go items significantly more when the Go item was remembered and the NoGo item was forgotten, versus when both were remembered or forgotten (see Fig. 4A), both for choices between high-value items (one-sided $P=0.026$, odds ratio $=2.258,95 \%$ CI $[0.993,5.135]$, mixed-effects logistic regression) and for choices between low-value items (one-sided $P=0.024$, odds ratio $=1.341,95 \% \mathrm{CI}[1.003,1.792])$, with a significant interaction between this accuracy category and the value category (onesided $P=0.040$ ). There were no significant differences in Go item choices when comparing pairs in which the Go item was forgotten and the NoGo item was remembered versus pairs in which both items were remembered or forgotten, neither for choices between high-value items (one-sided $P=0.102$ ) nor for choices between low-value items (one-sided $P=0.893$ ). The interaction effect was also not significant (one-sided $P=0.088$ ).

The relationship between the recognition $\Delta \mathrm{RT}$ and choices (see Fig. 5A) of Go over NoGo items was not significant, neither for high-value items (one-sided $P=$ 0.171 , mixed-effects logistic regression) nor for low-value items (one-sided $P=$ 0.302).

\section{Follow-up session (see Supplemental Table 5)}

Participants chose Go items more when the Go item was remembered and the NoGo item was forgotten, versus when both were remembered or forgotten (see Fig. 4B), when choosing between high-value items (one-sided $P<0.001$, odds ratio $=2.810,95 \%$ CI $[1.774$, 4.450], mixed-effects logistic regression), but not when choosing between lowvalue items (one-sided $P=0.237$ ), with a significant interaction between the accuracy category and the value category (onesided $P=0.002$, meaning that this effect was significantly stronger for high-value compared with low-value items). Again, there were no significant differences in Go item choices when comparing pairs in which the Go item was forgotten and the NoGo item was remembered versus pairs in which both items were remembered or forgotten, neither for choices between high-value items (one-sided $P=$ 0.589 , mixed-effects logistic regression) nor for choices between low-value items (one-sided $P=0.503$ ). The interaction effect was also not significant (one-sided $P=0.454$ ).

The relationship between the $\Delta \mathrm{RT}$ and choices (see Fig. 5B) of Go over NoGo items was not significant, neither for high-value items (one-sided $P=$ 0.174 , mixed-effects logistic regression) nor for low-value items (here the direction of the difference was negative; when the $\Delta \mathrm{RT}$ between the low-value NoGo and the low-value Go item was

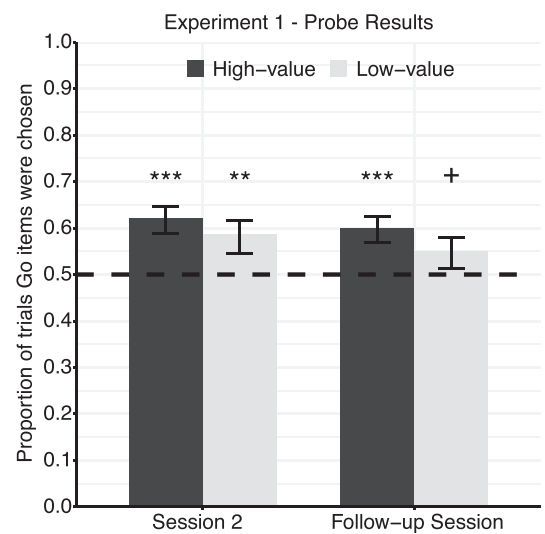

Figure 3. Probe results of experiment 1. Mean proportions of choosing the Go item (calculated for each participant and then averaged across participants) are presented with error bars representing standard error of the mean. Dashed line indicates 50\% chance level. Asterisks represent statistical significance of a one-sided logistic regression analysis; $(+) P<0.07$, ${ }^{(* *)} P<0.01,\left({ }^{* * *}\right) P<0.001$. 


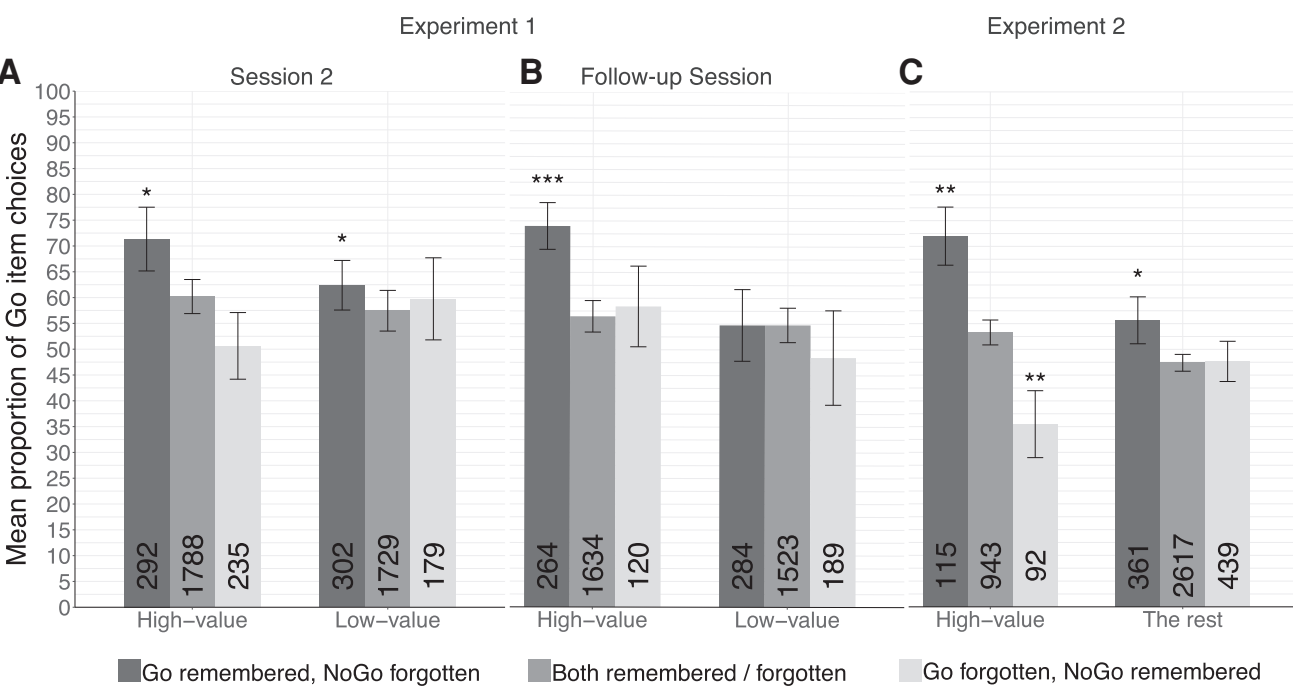

Figure 4. The relationships between recognition memory and choices in experiment 1 , session 2 (performed $3 \mathrm{~d}$ after session 1 ) ( $A$ ); experiment 1 , follow-up session ( $30 \mathrm{~d}$ after session 1$)(B)$; and experiment $2(C)$. Mean percent of Go item choices as a function of recognition memory with error bars representing standard error of the mean. The number of trials, summed across participants, is presented at the bottom of each bar. Asterisks represent statistical significance between each category and the "Both remembered/forgotten" baseline category (one-sided mixed-effects logistic regression). (*) $P<0.05,\left({ }^{*}\right) P<0.01,\left({ }^{* *}\right) P<0.001$.

larger, the Go item was less likely to be chosen; one-sided $P=$ $0.924)$. The interaction between the $\Delta \mathrm{RT}$ and value category was significant (one-sided $P=0.005$ ).

\section{Experiment 2}

The findings of experiment 1 demonstrated that, as predicted, memory was enhanced for Go compared with NoGo items following CAT. Furthermore, they suggested that choices following CAT were related to memory at the individual item level. To further examine these relationships and replicate the effect we found, in experiment 2 , we sought to increase the variance of memory scores across items and allow a better examination of the memory effects on individual items. To do so, we doubled the number of trained and tested items, and for the first time, we decreased the number of training repetitions to one. Our goal was to increase the variance across items, in pretraining preferences, post-training memory and post-training choices, to further examine the relationships between memory and value-based choices at the individual item level.

$N=35$ valid participants completed experiment 2, which was also preregistered. Three additional participants were excluded based on our preregistered exclusion criteria. Upon arrival, participants completed a BDM auction with 80 items (instead of 60 items

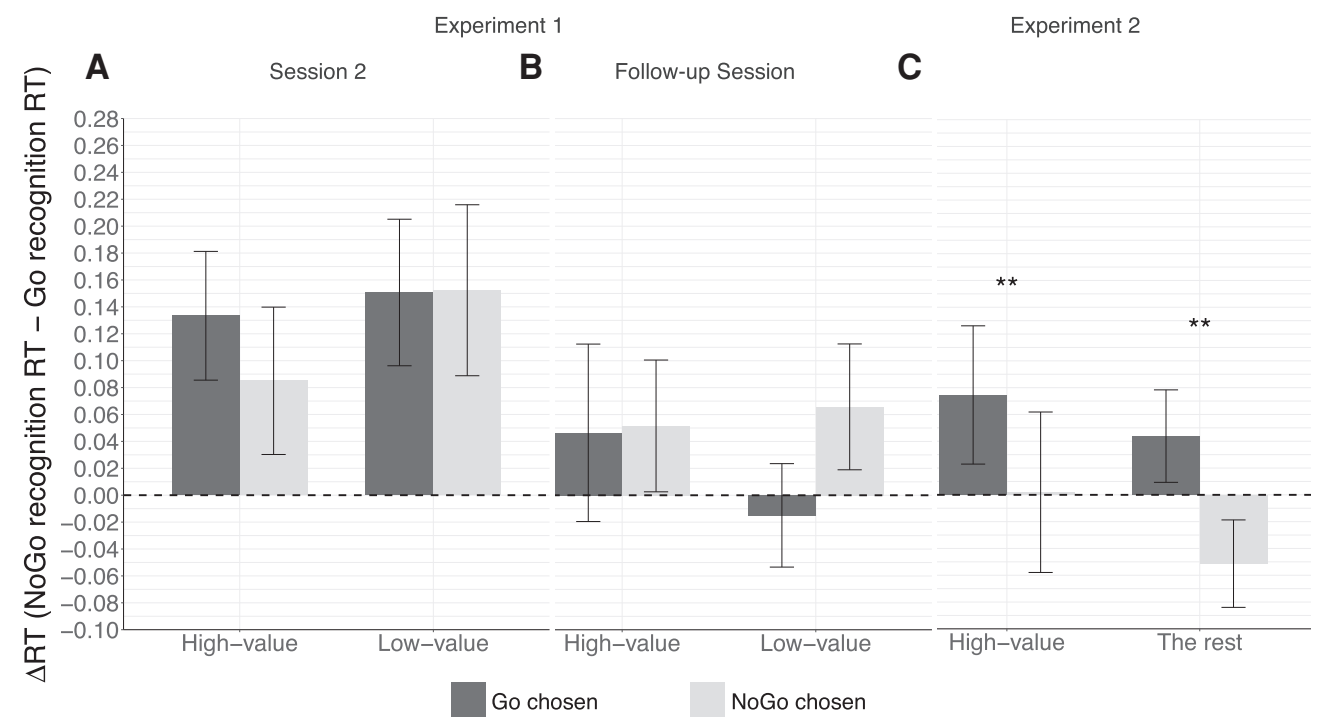

Figure 5. The relationships between recognition $\Delta R T$ and choices in experiment 1 , session 2 (performed $3 \mathrm{~d}$ after session 1) (A); experiment 1 , follow-up session (performed $\sim 30 \mathrm{~d}$ after session 1 ) (B); and experiment 2 (C). Mean $\triangle R T$ values (in seconds) are presented for choices of Go items and for choices of NoGo items, within each value category. Error bars represent standard error of the mean. Asterisks represent statistical significance of one-sided mixed-effects logistic regression; $\left({ }^{*}\right) P<0.01$. 
as in experiment 1), Then, they completed the training task, which was similar to experiment 1 , besides two important modifications: First, each item was only presented once (instead of 16 repetitions in experiment 1). Second, 80 (instead of 40) items were presented during training, with 12 high-value Go items and 12 low-value Go items (compared with six of each category in experiment 1). Following the training task, participants completed a filler task that lasted $\sim 30 \mathrm{~min}$. Then, their memory was tested with a recognition task, similar to the one performed in experiment 1 , but with a much larger total number of 160 items- 80 old and 80 new items. Finally, participants' preferences were revaluated with the probe task that included a doubled number of choices compared with experiment 1 .

\section{Recognition (see Supplemental Table 6).}

Overall performance. The mean hit rate of participants was $85.63 \%$ (SD $=11.34 \%)$. The mean correct rejection rate was $81.6 \%(\mathrm{SD}=$ $12.48 \%)$. The mean $d^{\prime}$ was $2.403(\mathrm{SD}=1.829)$. The mean $\mathrm{RT}$ was $1.384 \mathrm{sec}(\mathrm{SD}=0.214 \mathrm{sec})$ for hits, $1.558 \mathrm{sec}(\mathrm{SD}=0.290 \mathrm{sec})$ for misses, $1.436 \mathrm{sec}(\mathrm{SD}=0.239 \mathrm{sec})$ for correct rejections and 1.752 $\sec (\mathrm{SD}=0.349 \mathrm{sec})$ for false alarms.

Hit rate. In contrast to experiment 1 and to our preregistered prediction, in experiment 2 the hit rate in the old/new recognition task was not higher for Go $(M=84.67 \%)$ compared with NoGo $(M=85.78 \%)$ items (one-sided $P=0.759$, mixedeffects logistic regression) (see Supplemental Fig. 1a), but was significantly higher for higher value categories (one-sided $P=$ $0.004)$. There was no interaction between the value category (high/medium-high/medium-low/low value) and item type (Go/ NoGo; two-sided $P=0.570$ ).

Response times. Contrary to our predictions, RTs for correct responses were not significantly faster for Go $(M=1.380 \mathrm{sec})$ compared with NoGo $(M=1.383 \mathrm{sec}$ ) items (one-sided $P=0.484$, mixed-effects linear regression) (see Supplemental Fig. 1b), or significantly faster for higher value items (one-sided $P=0.211$ ). The interaction between item type and value category was not significant (two-sided $P=0.738$ ).

\section{Choices (for full statistics see Supplemental Table 7)}

In the high-value probe choices (equivalent to those of experiment $1)$, participants significantly chose Go over NoGo items $(M=$ $54.41 \%$, one-sided $P=0.035$, odds ratio $=1.209$, 95\% CI $[0.984$, 1.486], mixed-effects logistic regression; see Supplemental Fig. 2) after one training run. However, participants did not show preference modification for the Go items in the other value categories, or when combining the high and medium-high value categories to one category.

\section{Relationships between memory and choices (see Supplemental Table 8)}

In experiment 2 , we tested the relationships between choices and memory within each of the four value categories. Since preferences were only changed for high-value items, we combined the other three categories (i.e., "The rest") (see Supplemental Table 9 for statistics of each of these value categories) in our analysis. We tested the interactions between memory and value category by modeling the value category as high-value compared with the rest of the categories.

Participants chose Go items significantly more when the Go item was remembered and the NoGo item was forgotten compared with when both were remembered or forgotten (see Fig. 4C), both for choices between high-value items (one-sided $P=0.004$, odds ra- tio $=2.627$, 95\% CI $[1.275,5.410]$, mixed-effects logistic regression) and for choices between items in the rest of the value categories combined (one-sided $P=0.016$, odds ratio $=1.287,95 \%$ CI $[1.023,1.619])$. The interaction between this accuracy category and the value category (high-value compared with the rest) was significant (one-sided $P=0.016$ ). Participants chose Go items significantly less when the Go item was forgotten and the NoGo item was remembered, versus when both were remembered or forgotten (see Fig. 4C), when choosing between high-value items (one-sided $P=0.009$, odds ratio $=0.499,95 \%$ CI $[0.280,0.888]$, mixed-effects logistic regression), but not when choosing between items from the rest of the value categories (one-sided $P=0.270$ ). The effect was significantly stronger for choices between high-value items compared with choices between items from the rest of the value categories (an interaction effect; one-sided $P=0.009$ ).

The relationship between the $\Delta \mathrm{RT}$ and choices of Go over NoGo items (see Fig. 5C) was significantly positive for the highvalue items (one-sided $P=0.004$, odds ratio $=1.511$, 95\% CI $[1.108,2.059]$, mixed-effects logistic regression) as well as for the rest of the value categories combined (one-sided $P=0.002$, odds ratio $=1.371,95 \%$ CI $[1.101,1.708])$. The interaction between the $\Delta \mathrm{RT}$ and value category was not significant (one-sided $P=0.329$ ).

\section{Pilot experiment}

For full results of the pilot experiment, see "Pilot Experiment," Supplemental Figures 3-6, and Supplemental Tables 10-14 in the Supplemental Material. In contrast to our predictions and to dozens of previous samples with CAT (Schonberg et al. 2014; Bakkour et al. 2016, 2017, 2018; Veling et al. 2017; Zoltak et al. 2017; Salomon et al. 2018; Botvinik-Nezer et al. 2020), in the pilot experiment we did not find a significant probe effect of choosing high-value Go over high-value NoGo items. In addition, analysis of the recognition task revealed that like preferences, memory was also not enhanced for Go compared with NoGo items following CAT in this pilot sample. There are two main possible reasons why memory was not higher for Go compared with NoGo items in the recognition task. We found a considerable ceiling effect in the recognition task, where the mean hit rate across participants was $94.75 \%$. Importantly, we found that memory was related to the specific choices, as in experiments 1 and 2 . Furthermore, these relationships were mostly stronger for choices between high-value items compared with choices between low-value items.

\section{Exploratory analysis}

To further explore how choices are affected by memory for each alternative, we directly tested whether Go items were chosen more when the Go item was remembered and the NoGo item was forgotten versus when the Go item was forgotten and the NoGo item was remembered. With the combined data from our three experiments, we used the same mixed effects logistic model we used for the main probe analyses but added the accuracy category (Go remembered NoGo forgotten/Go forgotten NoGo remembered) as a predictor. Participants significantly chose Go items more when the Go item was remembered and the NoGo item forgotten compared with when the Go item was forgotten and the NoGo item was remembered, when choosing between high-value items (averaged ratio of Go choices: Go remembered NoGo forgotten $=66.15 \%$, Go forgotten NoGo remembered $=46.05 \%$; two-sided $P<0.001$, odds ratio $=2.653,95 \%$ CI $[1.540,4.570]$, mixed-effects logistic regression) but not when choosing between low-value items (averaged ratio of Go choices: Go remembered NoGo forgotten $=$ $56.08 \%$, Go forgotten NoGo remembered $=49.43 \%$; two-sided $P=$ 0.110 , odds ratio $=1.381,95 \%$ CI $[0.929,2.053]$, mixed-effects logistic regression). 


\section{Discussion}

The role of memory processes in value-based decision-making, as well as the cognitive and neural mechanisms by which memory influences decisions, are not yet fully understood (Shadlen and Shohamy 2016; Fellows 2017; Weilbächer and Gluth 2017; Biderman et al. 2020; Schonberg and Katz 2020). Recent studies have begun to highlight the interactions between memory and value-based decision-making. However, most of these studies involved external reinforcements, while everyday life involves decisions and associations that are not directly reinforced (Schonberg and Katz 2020).

Here, we tested the role memory processes play in the behavioral change of preferences following the CAT paradigm. This paradigm has been shown to reliably induce long-lasting preference change using the mere association of images of items with a neutral cue and a speeded button press response, without external reinforcements (Schonberg et al. 2014; Salomon et al. 2018, 2019; Botvinik-Nezer et al. 2020). We hypothesized that the cognitive mechanisms underlying the CAT effect are based on modifications of memory. We suggested that memory for Go items is enhanced following CAT. Therefore, we predicted better recognition memory for Go compared with NoGo items following CAT in the short and in the long term, and that the better memory of Go items is related to greater preferences for Go items with positive associative memories during probe.

\section{Experiment 1}

We tested our predictions in two preregistered experiments based on an additional pilot experiment. In experiment 1 , we tested these predictions with a recognition and a subsequent probe task, performed $3 \mathrm{~d}$ after CAT. Similar to previous studies with CAT (Schonberg et al. 2014; Bakkour et al. 2016, 2017, 2018; Veling et al. 2017; Zoltak et al. 2017; Salomon et al. 2018, 2019; Botvinik-Nezer et al. 2020), we found enhanced preference for Go items following CAT. High-value Go items were preferred over high-value NoGo items $3 \mathrm{~d}$ as well as 1 mo following CAT (Fig. 3). Low-value Go items were also preferred over low-value NoGo items. Experiment 1 was the first experiment with CAT in which choices were first tested $3 \mathrm{~d}$ (and not immediately) after training. In previous experiments, there was always a probe phase on the same day of training and then subsequent probes up to $6 \mathrm{mo}$ after the initial training. The results of experiment 1 show that training directly influences choices for at least a few days after training, therefore suggesting that the long-term duration of the CAT effect is less likely to be driven by previous choices via a mere choice effect (Sharot et al. 2012). A similar effect was recently shown 1 wk after Go/NoGo training irrespective of immediate choices (Chen et al. 2021).

As our suggested mechanism predicted, we also found a significant memory enhancement, reflected as higher hit rates and faster RTs in the recognition task for Go compared with NoGo items, both in the short and in the long term (Fig. 2). This is the first direct evidence of enhanced memory strength and accessibility for Go versus NoGo items following CAT, suggesting that memory modifications are involved in the underlying mechanism of the CAT effect. These findings are in accordance with recent studies showing enhanced recognition memory for Go compared with NoGo items in different versions of Go-NoGo and stop signal tasks (Chiu and Egner 2015a,b; Yebra et al. 2019). Most importantly, since we hypothesized that each choice is related to the memory for the specific items in the presented pair, we tested whether choices were related to the memory for the specific items on each binary choice pair. We found evidence showing that memory was indeed related to the specific choices. Choices of the Go items over the NoGo items were more likely when the individual Go item was remembered and the NoGo item was forgotten (Figs. 4, 5). Furthermore, these relationships were generally more positive for choices between high-value items compared with choices between low-value items. These results add to previous findings showing that enhanced preferences toward go items 1 wk after Go/NoGo training were related to immediate memory (but the memory was for the stimulus response contingencies rather for the items themselves) (Chen et al. 2021).

It should be noted that we originally planned to test recognition memory with paired $t$-tests and the relationships between memory and choices with linear correlation tests (see "Deviation from Preregistration"). The results of the $t$-tests are similar to the results of our reported results and are mostly significant (see the Supplemental Material, "Preregistered Analyses for Experiment 1 "). The results of the linear correlation tests are mostly insignificant. However, these linear correlations test the relationships between memory modifications and choices across participants, rather than across items, and are less suited compared with the tests we report here. The fact that correlation tests across participants yielded mostly insignificant results, while correlations across items yielded mostly significant results, further emphasize that CAT has an item-specific rather than an overall effect on items.

\section{Experiment 2}

The findings of experiment 1 suggested that CAT affects memory, and thus choices, at the individual item level. Therefore, in experiment 2 , we increased the number of items to more widely explore the variability of the effect across items. We also decreased the number of training repetitions to one, in order to test whether one run is sufficient to affect memory and preferences. We found that even a single training run was sufficient to induce preference modification in the highest value category tested (but not in other value categories). Overall, contrary to our preregistered predictions, memory was not significantly enhanced for Go compared with NoGo items. These results imply that one training run might be sufficient to change preferences for the most valued Go items (a change that was significant but weak compared with previous CAT samples), but not sufficient to induce an effect of enhanced memory across items.

Nonetheless, we again found significant relationships between memory and choices: Better-remembered Go items were more likely to be preferred over worse-remembered NoGo items in the binary probe phase, mainly for choices between high-value items (the value category in which the choice effect was found). These results provide additional evidence in support of our suggested mechanism, by replicating, in a different design, the finding that better-remembered Go items following CAT are also chosen more often. There are a few possible reasons for the absence of overall memory modifications, including the large number of items (80 items trained in experiment 2 vs. 40 in experiment 1 and the pilot experiment) and single training repetition (which were very different from the standard CAT paradigm), or a lack of consolidation in such short time scales (memory was tested $\sim 30$ min after CAT).

\section{Pilot experiment}

Prior to these two experiments, we conducted a pilot experiment (see the Supplemental Material), which was similar to experiment 1 , but with an additional probe task performed at the end of session 1. In this pilot sample, we also found positive relationships between memory for individual Go items and choices of these items over worse remembered NoGo items. Unlike previous studies, there was no overall CAT effect in the pilot experiment. We cannot explain why the CAT effect did not replicate in this specific pilot sample. We did not find differences between the current sample 
and previous ones, with regard to demographic information, BDM ratings, performance during training or any other aspect of the task. We also did not find enhanced memory for Go compared with NoGo items, possibly due to a ceiling effect in recognition memory performance in this experiment. Nevertheless, the existence of a link between memory and choices at the individual item level in the pilot experiment indicates that even when the main effects of both choices and memory are absent, individual choices are still related to memory for the individual items.

\section{Exploratory analyses and alternative explanations}

To further explore the relationship between memory and choices, we directly tested whether choices of Go items were more likely when the Go item was remembered and the Go item forgotten compared with when the Go item was forgotten and the NoGo item was remembered. Using the combined data from the three experiments, we found that it was indeed the case for choices between high-value items, but not for choices between low-value items (see "Supplemental Exploratory Analyses" in the Supplemental Material). This finding is predicted by our suggested model, since choices of better remembered items are expected when the items are valued, and not when they are not (in such a case, associative memories could be negative and therefore may lead to choosing the other option).

We interpreted faster RTs for Go compared with NoGo items in experiment 1 as an indication of better memory for Go items, in accordance with our preregistered hypotheses and predictions. However, an alternative explanation for this finding might be that the association of Go items with motor responses during training resulted in a conditioned response of pressing when Go items are presented, leading to faster RTs for these items irrespective of memory strength. To disentangle these two hypotheses, we performed exploratory analyses on the recognition data from experiment 1 (see "Supplemental Exploratory Analyses" in the Supplemental Material). The results of this analysis suggest that the faster RTs for Go compared with NoGo items in the old/new recognition task are indeed an indication of better memory for these items, and not of a response bias.

Response bias can also putatively explain the interactions we found between recognition memory and choices following CAT, leading participants to respond affirmatively to Go items in both the memory recognition and the binary-choice probe tasks. However, findings of the current as well as previous studies do not support this possibility. First, CAT affects choices between high-value items but frequently not choices between low-value items (Schonberg et al. 2014; Botvinik-Nezer et al. 2020). A response bias would have been predicted to also affect low-value choices. Moreover, the CAT effect was also found in a previous sample when choices were made with the eyes rather than the hands (Bakkour et al. 2016), further indicating that the effect of CAT is not solely a response bias in the hand motor circuit.

Another alternative explanation for the interactions we found between memory and choices following CAT, is that CAT influences a third variable, such as familiarity, which affects both choices and memory. However, all items included in our stimuli data set are local familiar snack food items, and our item allocation procedure, which matches choice pairs based on the initial WTP, ensures that any pre-CAT difference between Go and NoGo items would be random. Furthermore, the number of times each item is presented during CAT is the same for Go and NoGo items; therefore, a mere exposure effect cannot explain the CAT effect (Zajonc 1968). Nevertheless, the current study did not explicitly manipulate familiarity, and future studies can test this alternative explanation, for example by comparing familiar with unfamiliar items (e.g., local snacks vs. snacks from other countries, or familiar vs. unfamil- iar faces). Another third variable might be emotional devaluation toward NoGo items as a result of inhibition (Fenske and Raymond 2006; Driscoll et al. 2018). However, it is not clear whether emotional devaluation would lead to the observed memory differences and correlations with choices. This can also be directly tested in future studies.

\section{Differential effect on high-value compared with low-value items}

An interesting aspect of CAT with snack food items is that it overall affects choices of high-value Go over NoGo items more than choices of low-value Go over NoGo items, mainly with snack food items (Schonberg et al. 2014; Salomon et al. 2018; Botvinik-Nezer et al. 2020). This effect was suggested to indicate that CAT may induce value scaling, rather than a fixed-increment value enhancement (McGuire and Kable 2014). Alternatively, we suggest that this differential effect is due to the greater number of positive memories associated with high-value versus low-value snacks. Our proposed mechanism suggests that memory is enhanced for both high-value and low-value Go items following CAT. When presented with binary choices of high-value items, the memories associated with Go items that are activated are usually positive, resulting in a preference for these items. When choosing between low-value items, activated associations are not necessarily positive since these items are subjectively less preferred to begin with (but are still mostly liked). Therefore, choices of low-value Go over low-value NoGo items following CAT are less consistent.

Unlike previous studies with CAT and snack food items, here we did not find a significant differential effect, in which the proportion of Go items choices is higher for high-value versus lowvalue items. However, it should be noted that in the current study we used a different and putatively improved random effects structure. Results were more similar to previous studies when using the same models as in our older work. This suggests that the variance was likely underestimated in some of the previous results and explains why we did not replicate some of the previous findings in this work, with the new mixed-effects models. Nonetheless, since it is likely that some of the low-value snack food items in the current and other experiments with CAT were liked by the participants while some where not (with individual differences across participants), predictions as well as results with regard to choices between low-value items are less straightforward than high-value items.

Overall, the findings of the current study were in line with some of our preregistered hypotheses. With the standard CAT paradigm, when choices were affected, memory was enhanced for Go compared with NoGo items. This enhancement occurred irrespective of value category (low-value or high-value), suggesting that, as expected, there is no differential effect of CAT on memory (i.e., that memory enhancement is not stronger for high-value Go compared with low-value Go items). Most importantly, when examining memory for each of the individual items in binary choices, we found that better memory for the Go compared with the NoGo items was related to choices of Go over NoGo items, more for highvalue compared with low-value choice pairs.

\section{Limitations}

It should be noted that the current study tested and showed that high-value remembered Go items were chosen over high-value forgotten NoGo items, and not that remembered high-value items were chosen over forgotten high-value items (i.e., remembered high-value NoGo items were not always chosen over forgotten high-value Go items) (see Fig. 4; Supplemental Fig. 5). This finding 
indicates that memory is not the only process underlying the CAT effect.

It is also important to note that our findings are correlational and do not establish a causal effect of memory on choices following CAT. This could have been further tested with a mediation analysis, however unfortunately our design is not suitable for this analysis. Future studies can be designed to test the causal effect of memory on choices in a more direct way. As memory was shown to be affected by preferences in previous studies, we cannot rule out the possibility that preference led to memory enhancement rather than the other way around.

\section{Related theories and frameworks}

Our suggested mechanism and our findings, emphasizing the involvement of memory processes in choices, are in line with a few previous theories, such as the preferences as memories framework (PAM) (Weber and Johnson 2006) and the query theory (Johnson et al. 2007). According to the PAM framework, preferences are the product of memory processes, such that relevant knowledge (rather than some kind of stored "value") is retrieved from memory when choice alternatives are compared, and thus choices are highly affected by basic memory processes such as priming and reactivation (Weber and Johnson 2006). The query theory (Johnson et al. 2007) also suggests that preferences are constructed at the time of choice, rather than stored and then retrieved during choices. Query theory further suggests that choices are made based on answers to questions, or queries, which are internally raised sequentially. According to this theory, the order of these queries, which is dependent on the context at the time of choice, substantially affects choices (Johnson et al. 2007). In the context of CAT, better remembered Go items may drive the order of internal queries, such that queries in favor of these items arise first and therefore drive the choice process toward Go items.

Another line of research related to our findings is "memory bias" that have been found in decisions from memory, such that people prefer remembered over forgotten options, even if remembered options are worse than average (unless remembered options are very unattractive) (Gluth et al. 2015). It has been shown that this memory bias is the result of people's beliefs that their memory is stronger for better compared with worse options (i.e., that a good option is remembered because it is good, while memory of a bad option is not related to its attractiveness) (Mechera-Ostrovsky and Gluth 2018). These findings may explain the results of the current study, raising the possibility that better remembered highvalue items are chosen more because participants attribute the better memory of these items to their value, while the memory for better-remembered low-value items is not attributed to value (or even attributed to their unattractiveness, and thus they are not chosen). However, these studies used tasks in which items were paired with specific locations on the screen, and then during the time of choice, these specific locations were presented without the items. Hence, the choice task in those studies forced participants to retrieve the options from memory, and a forgotten option was unknown during choices. This is in contrast to the binary choice task used in CAT, where both items are presented at the time of choice, and the memory measure is related to remembering that a specific item appeared during training.

Our hypothesized mechanism suggests that items with greater memory accessibility are chosen more. One possible mechanism for this process was recently proposed (Shadlen and Shohamy 2016; Bakkour et al. 2019) based on the observation that harder decisions (e.g., between options with similar subjective values) take longer (Krajbich et al. 2010; Milosavljevic et al. 2010). In perceptual decision-making, perceptual evidence accumulates with time and a decision is made once a given threshold of evidence has been reached (Gold and Shadlen 2007; Shadlen and Kiani 2013). However, during value-based decision-making, it is unclear what consumes the time to decision. Shadlen and Shohamy (2016) proposed that these decisions are based on retrieval of accumulated evidence from episodic memory. In the framework of evidence accumulation from episodic memory during value-based decisionmaking, the relationships we found between choices and memory may be driven by a faster rate of evidence accumulation from memory in favor of the better remembered Go items. It should be noted, however, that while we did find evidence for correlations between both memory strength (accuracy) and accessibility (response rate) and choices, our findings were overall stronger for memory strength compared with memory accessibility.

If choices are indeed affected by the rate of accumulation from memory as suggested by Shohamy and Shadlen's model (Shadlen and Shohamy 2016), choices of remembered items should be faster compared with choices of forgotten items. To test this prediction, we performed exploratory analysis (see "Supplemental Exploratory Analyses" in the Supplemental Material). In support of this potential mechanism, we found that choices were significantly faster when the chosen item was remembered compared with when it was not remembered. Future studies could be designed to further explore Shohamy and Shadlen's suggested model (Shadlen and Shohamy 2016) in the framework of CAT and beyond.

\section{Conclusions}

In conclusion, our findings support our suggested mechanism underlying the CAT effect, by showing that memory is enhanced for trained items and that enhanced memory is related to subsequent choices. These findings align with a growing literature showing diverse interactions between memory and choices. Nonetheless, to the best of our knowledge this is one of the first demonstrations of the involvement of memory in preference change in the absence of external reinforcements. These results shed light on the mechanism underlying behavioral change following CAT, which offers novel avenues for long-lasting behavioral change interventions for maladaptive behaviors such as eating disorders and addictions.

\section{Materials and Methods}

\section{Data sharing and preregistration}

All of our data and codes are publicly shared on GitHub (https://doi .org/10.5281/zenodo.5008851, release 3.0.0). Preregistrations can be found on the Open Science Framework (OSF; experiment 1: https://doi.org/10.17605/OSF.IO/V79MS; experiment 2: https ://doi.org/10.17605/OSF.IO/YH749).

\section{Participants}

A total of $N=95$ participants took part in this study, each completed one of the three experiments-experiment $1 \quad(n=35)$, experiment $2(n=35)$, or a pilot experiment $(n=25)$. See Table 1 for a demographic description of each experimental sample, and the Supplemental Material for a detailed description of the pilot experiment. We preregistered the sample sizes of experiment 1 and experiment 2 . A power analysis performed with our previous CAT samples, yielded a minimal $n=20$ for detecting the choice effect with $80 \%$ power and $\alpha=0.05$. However, since the current experiments focused on memory, which was not similarly tested before with CAT, and also taking into account that some participants would probably not return to the follow-up session, we chose to preregister a larger sample size of $n=35$ for each experiment.

All participants had normal or corrected-to-normal vision and hearing and provided signed informed consent to participate in the experiment in return for monetary compensation. Participants were asked to refrain from eating and drinking 
anything but water for $1 \mathrm{~h}$ prior to each visit in the laboratory. The study was approved by the ethics committee of Tel Aviv University.

\section{Stimuli}

Stimuli in this study comprised of colored images of familiar local Israeli snack food items. Images depicted the snack package and the snack itself on a homogenous black rectangle sized $576 \times 432$ pixels. The stimuli were created in our laboratory and are available online (http://schonberglab.tau.ac.il/resources/snack-food-imagedatabase). To promote incentive compatible behavior, when participants entered the laboratory, they were presented with a cabinet containing the real snacks and the items were available for actual consumption at the end of the experiment.

\section{Procedures}

The experiment was run on a 21.5-in iMAC computer with Matlab version 2014b (Mathworks, Inc.), the Psychtoolbox (http://www .psychtoolbox.org) and Python-based Pygame package (Python version 2.7).

The general design included an auction (used to obtain subjective willingness to pay), training, a recognition task and a binary choice task (see Fig. 1). The tasks, besides the recognition task, were similar to previous studies with CAT (Schonberg et al. 2014; Salomon et al. 2018). The variants of the design for each experiment are described below.

\section{Subjective preferences evaluation (see Fig. 1A)}

First, subjective preferences for the snack food items (60 items in experiment 1 and the pilot experiment, and 80 items in experiment 2) were evaluated using a Becker-DeGroot-Marschak (BDM) auction procedure (Becker et al. 1964). Participants were first endowed with 10 Israeli shekels (ILSs; equivalent to $\sim 2.7 \$$ US). During the auction, snack food items were individually presented on the screen in a random order. The task was self-paced. Participants were asked to indicate how much money they are willing to pay for each item, using the mouse cursor along a continuous visual analog scale, ranging from 0-10 ILSs. Participants were informed in advance that at the end of the experiment, the computer will randomly choose one of the items and will generate a counter bid. In case the bid placed by the participant exceeds the computer's bid, she or he will be required to buy the item for the computer's lower bid price. Otherwise, the participant will not buy the item and will keep the allocated 10 ILSs. Instructions explicitly mentioned that the best strategy for this task was to indicate the actual willingness to pay (WTP) for each item.

\section{Item selection}

For each participant, items were rank-ordered based on the subjective WTP values. Items were split to high-value (above the median) and low-value (below the median) items. One set of items within each value category was used as Go items, while another set of items, with identical mean ranks, was used as NoGo items (see Supplemental Table 1). These sets of items were counter-balanced across participants. This item selection procedure allowed us to present choices of high-value Go versus high-value NoGo items, as well as choices of low-value Go versus low-value NoGo items, with similar initial WTPs, in the probe binary phase. Of the items presented during training, 30\% were Go items (12 out of the 40 items in experiment 1 and the pilot experiment; 24 out of the 80 items in experiment 2).

\section{Training (see Fig. 1B)}

During the training session, individual items were presented on the screen one by one for $1 \mathrm{sec}$ each. We instructed participants to press the " $\mathrm{b}$ " button on the keyboard as fast as they could whenever an auditory Go cue was heard. Unbeknownst to participants, the cue was consistently paired with Go items and not with NoGo items. The neutral auditory cue, consisted of a 180-msec-long sinus wave function, was initially heard $750 \mathrm{msec}$ after the stimulus on- set. Aiming for a success rate of $\sim 75 \%$ (successful button press before the image disappeared from the screen), we updated the delay between the stimulus onset and cue onset with a ladder technique, such that the delay was increased by $16.67 \mathrm{msec}$ following a successful trial and decreased by $50 \mathrm{msec}$ following a missed trial. We used a jittered interstimulus interval (ISI) from a truncated exponential distribution, ranging from 1 to $6 \mathrm{sec}$ (1-sec interval) and an average duration of 2 sec. Importantly, no external reinforcement was provided to the participants with regard to their performance on the task.

\section{Recognition (see Fig. 1C)}

The recognition task was added to the paradigm in order to test our proposed mechanism. The recognition task was performed either $\sim 30$ min after training (experiment 2 ) or $3 \mathrm{~d}$ and again 1 mo following training (experiment 1). In this task, all items that would be included in the subsequent Go-NoGo binary choice probe pairs, as well as an equal number of new items, were presented on the screen one at a time. Participants responded for two consecutive questions: (1) Was the item presented during the experiment? (2) Was the item paired with a cue during training? Participants responded using a five-point confidence scale (1-sure yes, 2 - think yes, 3-uncertain, 4-think no, 5-sure no) within a maximal time frame of $3 \mathrm{sec}$.

\section{Probe (see Fig. 1D)}

In the probe phase, we tested participants' preferences following CAT, using a binary choice task. On each trial, participants were presented with a pair of items from the same value category (both either high-value or low-value), one Go item (i.e., consistently paired with the cue and button press during training) and one NoGo item (i.e., not paired with the cue and button press during training). On each trial, participants were given $1.5 \mathrm{sec}$ to choose the item they preferred, by pressing the button " $u$ " (to choose the left item) or the button "i" (to choose the right item) on the keyboard. Participants were told in advance that at the end of the experiment, one trial will be randomly drawn by the computer and that they will receive the item they chose on the randomly drawn trial. The ISIs were sampled from a truncated exponential distribution, ranging from 1 to $12 \mathrm{sec}(1-\mathrm{sec}$ interval) and an average duration of 3 sec.

\section{Exclusion criteria}

Participants were excluded from the final analysis if they placed bids lower than 1 ILS ( $~ \$ 0.27$ US) on $>40$ items during the BDM auction (Schonberg et al. 2014; Salomon et al. 2018); reached a ladder $<200$ msec at least once during training, pressed the button without a cue on $>5 \%$ of NoGo training trials $(>5 \%$ false alarm rate) and/or did not press the button following a cue on $>10 \%$ of Go training trials (>10\% misses). These exclusion criteria were listed in the preregistration of experiments 1 and 2.

\section{Analysis}

All analyses were performed using $\mathrm{R}$ version 3.6.3. For all mixed-effects models, we used R's glmer and lmer functions (with the packages "Ime4" [Bates et al. 2015] and "ImerTest" [Kuznetsova 2017]). In all models, random effects were modeled within participants (i.e., we included a random intercept and random slope term per participant). In each model we started with a maximal random effects structure, modeling all random effects and their correlations (Barr et al. 2013). In case the model did not converge properly, we simplified the maximal model by first removing the random correlations and then reducing the random terms that indicated model converges issues (i.e., correlations of 1 , or random variance of 0 ). Using these criteria preserves type I error while potentially increasing power when random effects estimates are near the boundary values (Matuschek et al. 2017). 


\section{Recognition}

We predicted that Go stimuli will be better remembered compared with NoGo items (regardless of value category), reflected in higher accuracy (hit rate) and faster response times (RTs) in the old/new recognition task. We only included items that were presented during the probe task in pairs comparing high-value Go versus highvalue NoGo or low-value Go versus low-value NoGo items, to ensure that both the number and the mean rank of Go and mean rank of NoGo items within each value category are equal.

Responses in the recognition task were transformed to a binary scale (correct/incorrect), with "uncertain" responses counted as incorrect answers in order to filter out correct guesses. Missed responses were excluded from analysis. Then, we tested the prediction regarding the hit rate with a one-sided mixed-effects logistic regression with the outcome (correct/incorrect) as the dependent variable and the item type (Go/NoGo) and value category (highvalue/low-value) as independent variables. The prediction regarding the RTs was tested with a one-sided mixed-effects linear model with the RT as the dependent variable and the item type (Go/ NoGo) and value category (high-value/low-value) as independent variables. In the RT analysis, we included only correct responses, as faster RTs for wrong answers do not imply better memory.

To test for the differential effect at the level of memory modification (i.e., whether Go items are better remembered over NoGo items to a greater extent in pairs of high-value compared with lowvalue items), in a hierarchical regression, we tested the significance of the interaction between item type (Go/NoGo) and value category (high-value/low-value).

\section{Choices}

We predicted that the CAT effect observed in previous studies will be replicated, such that participants will significantly choose high-value Go over high-value NoGo items (Schonberg et al. 2014; Bakkour et al. 2017; Veling et al. 2017; Salomon et al. 2018; Botvinik-Nezer et al. 2020). Similar to previous studies with the cue-approach task, we tested this prediction using a onesided mixed-effects logistic regression, comparing the odds of choosing Go items against chance level (log odds $=0$; odds ratio $=$ 1 ), independently for each value category (high-value and lowvalue). An interesting aspect of CAT, demonstrated in previous studies with snack food items, was that its effect is greater in choices of high-value Go over NoGo items, than in choices of lowvalue Go over NoGo items (Schonberg et al. 2014; Salomon et al. 2018; Botvinik-Nezer et al. 2020). Therefore, similar to previous studies, we further tested whether there was a differential effect of higher proportions of Go items choices on high-value compared with low-value probe pairs, with a one-sided mixed-effects logistic regression.

\section{Relationships between memory and choices}

We hypothesized that Go items are preferred over NoGo items because they are more accessible in memory, and therefore their associations accumulate faster to choices and they are chosen more when their associations are positive. Therefore, we predicted that binary choices will be related to memory for the specific alternatives on a trial-by-trial basis, such that better memory for the Go compared with the NoGo item will be related to more Go item choices, mainly for high-value items.

We tested this prediction with two mixed-effects logistic models predicting choices of Go items based on the old/new recognition results: (1) The accuracy model combined the memory for the Go item and the memory for the NoGo item to an independent variable with three accuracy categories: "Go remembered and NoGo forgotten," "Both remembered/forgotten," and "Go forgotten and NoGo remembered" (pairs in which both items were remembered were combined with pairs in which both items were forgotten since we did not have explicit predictions about differences between these categories, and also because the number of pairs with two forgotten items was relatively small in most of our analyses). (2) The RT model included one independent variable, the $\Delta R T$ (RT NoGo - RT Go; only for probe trials in which both items were correctly remembered).

According to our suggested mechanism, better remembered items should be preferred over worse remembered items when associated memories are positive. Therefore, we predicted that this relationship will be stronger for high-value compared with low-value items. We tested this prediction with a mixed-effects logistic regression model with the main effect of value category (high-value/lowvalue) and the interaction between the value category and the recognition variables. Participants were modeled as a random effect in all models.

\section{Exploratory analyses}

In addition to our preregistered analyses, we performed the following exploratory analyses (see the Supplemental Material for 2-5): (1) directly comparing the ratio of choosing Go items when the Go item is remembered and NoGo item is forgotten versus when the Go item is forgotten and the NoGo item is remembered, (2) comparing RT between Go and NoGo items for incorrect responses (Misses) in order to validate our RT memory effects, (3) comparing hit rate and RTs between Go and NoGo items in the recognition Go/NoGo task, (4) comparing the confidence levels in the old/ new recognition task between Go and NoGo items, and (5) comparing choice RT for remembered versus forgotten items and for Go versus NoGo items.

\section{Deviation from preregistration}

It should be noted that the statistical tests used were correctly preregistered for experiment 2 but not for experiment 1 , where we preregistered we will use $t$-tests and correlation tests in the recognition analysis and in the analysis of relationships between memory and choices, respectively. However, prior to data analyses, we decided to use the more appropriate mixed-effects tests that are described above (and were preregistered for experiment 2 , during data collection of experiment 1 ). Nonetheless, paired $t$-tests and linear correlations results are reported in the Supplemental Material (see section "Preregistered Analyses for Experiment 1"). We also note that our preregistered analysis plan was not always detailed enough, and some of the models were not explicitly described in enough detail. For example, although our prehypothesized suggested mechanism predicts that better remembered items are chosen more when their associations are positive, we did not explicitly include the predictions of the differences between highvalue and low-value items in our preregistered analysis plan. These predictions were made prior to analysis and unintentionally their full details were not described in the preregistration. Furthermore, in experiment 2 we analyzed the results based on four value categories (high/medium-high/medium-low/low), although this split was not mentioned in our preregistration, but was used in the original CAT studies (Schonberg et al. 2014). In addition, the preregistered plan included eye-tracking analysis. While eye-tracking data were indeed collected for some of the participants for exploratory purposes, these data are beyond the scope of this paper, were not analyzed in this study and are not presented here.

\section{Experiment 1}

In experiment 1 , we added a recognition task $3 \mathrm{~d}$ following CAT (i.e., in session 2). The recognition task was added $3 \mathrm{~d}$ and not immediately following CAT to avoid a ceiling effect that was present in previous experiments in our laboratory when the recognition task was performed immediately following the probe task (Salomon et al. 2018; Botvinik-Nezer et al. 2020).

Thirty-five valid participants completed the first two sessions, of them $n=30$ returned for an additional follow-up session, $\sim 1 \mathrm{mo}$ after session $1(M=34.3 \mathrm{~d}, \mathrm{SD}=5.04 \mathrm{~d}$, range $=28-46 \mathrm{~d})(\mathrm{demo}-$ graphic statistics are reported in Table 1). Four additional participants were excluded based on preregistered exclusion criteria: Two bid less than one ILS on $>40$ items (BDM exclusion criteria) and two reached a ladder $<200 \mathrm{msec}$ during training and also pressed the button when not needed (false alarms) on $>10 \%$ of 
training trials. One participant completed only one of the two probe blocks (i.e., one instead of two repetitions of each probe pair). This participant was included in the analysis; however, the results do not change when this participant is excluded from the analysis.

\section{Session 1}

Upon arrival, we evaluated participants' initial preferences for 60 snack food items with a BDM auction (Fig. 1A). They then completed 16 repetitions of CAT. In each training repetition, 40 items were presented in a random order (Fig. 1B).

\section{Session 2}

Exactly $3 \mathrm{~d}$ after session 1, participants returned to the laboratory for session 2. They completed the recognition task (Fig. 1C), probe task (Fig. 1D) and an additional BDM auction (Fig. 1A).

The recognition task included 56 items: 28 new items that were not previously presented during the experiment and 28 old items that were previously presented during the experiment (these would later be used in the probe phase: 24 stimuli in the Go vs. NoGo pairs and four additional items from the "sanity check" probe pairs).

Following the recognition task, modifications of preferences were evaluated in a probe task. The probe task consisted of a set of binary choices, in which each of the six high-value Go items was pitted against each of the six high-value NoGo items $(6 \times 6=$ 36 comparisons) and each of the six low-value Go items was pitted against each of the six low-value NoGo items $(6 \times 6=36$ comparisons). Thus, overall, there were 72 unique pairs of Go versus NoGo item comparisons. We also included "sanity check" probe pairs, in which high-value NoGo items were pitted against lowvalue NoGo items. Each unique comparison was repeated twice during the experiment (once in each of two task blocks), resulting in overall 144 Go-NoGo probe trials.

\section{Follow-up session}

About 1 mo after session 1, participants returned to the laboratory and completed the follow-up session, which included the same tasks as in session 2 .

\section{Experiment 2}

Experiment 2 included $n=35$ valid participants. Three additional participants were excluded based on the preregistered BDM exclusion criteria. Upon arrival, participants completed a BDM auction with 80 items (instead of 60 items as in experiment 1), Then, participants completed the training task, which was similar to experiment 1, besides two important modifications: First, each item was only presented once (instead of 16 repetitions in experiment 1 ). Second, 80 (instead of 40) items were presented during training. Out of the 80 items, 12 items were high-value Go items and 12 items were low-value Go items (compared with six high-value Go and six low-value Go items in experiment 1).

After training, participants completed a filler task during which they ranked fractal art images, unfamiliar faces and familiar faces (of local politicians), as well as the familiarity of the familiar faces. The filler tasks lasted $\sim 30 \mathrm{~min}$. Following the filler tasks, participants completed a recognition task, similar to the one performed in experiment 1, but with a larger total number of 160 items: 80 old and 80 new items.

Then, participants completed the probe task. Since we doubled the number of trained items, we also doubled the number of probe comparisons. Probe Go-NoGo choices included four value categories: high, medium-high, medium-low and low value (for the exact ranks of each value category, see Supplemental Table 1). Each category included 36 unique choices between the six Go items pitted against six NoGo items of identical mean rank. Thus, overall, each probe block included a total of 144 unique Go versus NoGo choices. Twelve "sanity check" probe trials (i.e., choices between high-value and low-value NoGo items) were also presented, as in experiment 1.

Finally, participants completed a familiarity task for the items, in which they ranked the familiarity of each snack food item. However, the results of this task are beyond the scope of this study.

\section{Pilot experiment}

Prior to experiments 1 and 2, we collected a pilot sample. The pilot experiment was similar to experiment 1 with one important difference: Participants also completed an additional probe task at the end of session 1. After exclusion of two participants based on the BDM exclusion criteria, the pilot experiment included $n=25$ valid participants (14 of them completed the follow-up session). For full details about the pilot experiment, see the Supplemental Material.

\section{Competing interest statement}

The authors declare no competing interests.

\section{Acknowledgments}

We thank Dr. Jeanette Mumford for her invaluable statistical advice. We thank Alex Manevich, Neta Cohen, and Michal Teitler for helping with data collection. R.B.-N. is an Awardee of the Weizmann Institute of Science-Israel National Postdoctoral Award Program for Advancing Women in Science. This work was supported by a grant from the European Research Council (ERC) under the European Union's Horizon 2020 research and innovation programme (grant agreement no. 715016) and a grant from the Israel Science Foundation (ISF), both granted to T. Schonberg. R.B.-N. thanks the Naomi Foundation through the Tel Aviv University Global Research and Training Fellowship Program for their support. A.B. was supported by a fellowship from the National Science Foundation (NSF; SBE Postdoctoral Research Fellowship grant no. 1606916). T. Salomon was supported by the Nehemia Levtzion fellowship and the Fields-Rayant Minducate Learning Innovation Research Center.

\section{References}

Anderson JR. 1983. A spreading activation theory of memory. J Verbal Learning Verbal Behav 22: 261-295. doi:10.1016/S0022-5371(83) 90201-3

Bakkour A, Leuker C, Hover AM, Giles N, Poldrack RA, Schonberg T. 2016. Mechanisms of choice behavior shift using cue-approach training. Front Psychol 7: 421. doi:10.3389/fpsyg.2016.00421

Bakkour A, Lewis-Peacock JA, Poldrack RA, Schonberg T. 2017. Neural mechanisms of cue-approach training. Neuroimage 151: 92-104. doi:10 .1016/j.neuroimage.2016.09.059

Bakkour A, Botvinik-Nezer R, Cohen N, Hover AM, Poldrack RA, Schonberg T. 2018. Spacing of cue-approach training leads to better maintenance of behavioral change. PLOS ONE 13: e0201580. doi:10 .1371 /journal.pone.0201580

Bakkour A, Palombo DJ, Zylberberg A, Kang YHR, Reid A, Verfaellie M, Shadlen MN, Shohamy D. 2019. The hippocampus supports deliberation during value-based decisions. Elife 8: e46080. doi:10.7554/ eLife. 46080

Barr DJ, Levy R, Scheepers C, Tily HJ. 2013. Random effects structure for confirmatory hypothesis testing: keep it maximal. J Mem Lang 68: 255278. doi:10.1016/j.jml.2012.11.001

Bates D, Mächler M, Bolker BM, Walker SC. 2015. Fitting linear mixed-effects models using lme4. J Stat Softw 67. doi:10.18637/ jss.v067.i01

Becker GM, DeGroot MH, Marschak J. 1964. Measuring utility by a single-response sequential method. Behav Sci 9: 226-232. doi:10.1002/ bs.3830090304

Bhatia S. 2013. Associations and the accumulation of preference. Psychol Rev 120: $522-543$. doi: $10.1037 / \mathrm{a} 0032457$

Biderman N, Bakkour A, Shohamy D. 2020. What are memories for? The hippocampus bridges past experience with future decisions. Trends Cogn Sci 24: 542-556. doi:10.1016/j.tics.2020.04.004

Bornstein AM, Norman KA. 2017. Reinstated episodic context guides sampling-based decisions for reward. Nat Neurosci 20: 997-1003. doi:10 $.1038 /$ nn. 4573 
Bornstein AM, Khaw MW, Shohamy D, Daw ND. 2017. Reminders of past choices bias decisions for reward in humans. Nat Commun 8: 15958. doi:10.1038/ncomms15958

Botvinik-Nezer R, Salomon T, Schonberg T. 2020. Enhanced bottom-up and reduced top-down fMRI activity is related to long-lasting nonreinforced behavioral change. Cereb Cortex 30: 858-874. doi:10.1093/cercor/ bhz132

Chammat M, El Karoui I, Allali S, Hagège J, Lehongre K, Hasboun D, Baulac M, Epelbaum S, Michon A, Dubois B, et al. 2017. Cognitive dissonance resolution depends on episodic memory. Sci Rep 7: 41320. doi: $10.1038 /$ srep4 1320

Chen Z, Holland RW, Quandt J, Dijksterhuis A, Veling H. 2021. How preference change induced by mere action versus inaction persists over time.Judgm Decis Mak 16: 201-237.

Chiu XY, Egner T. 2015a. Inhibition-induced forgetting results from resource competition between response inhibition and memory encoding processes. J Neurosci 35: 11936-11945. doi:10.1523/ JNEUROSCI.0519-15.2015

Chiu Y-C, Egner T. 2015b. Inhibition-induced forgetting: when more control leads to less memory. Psychol Sci 26: 27-38. doi:10.1177/ 0956797614553945

Driscoll RL, De Launay KQ, Fenske MJ. 2018. Less approach, more avoidance: response inhibition has motivational consequences for sexual stimuli that reflect changes in affective value not a lingering global brake on behavior. Psychon Bull Rev 463-471. doi:10.3758/ s13423-017-1291-y

DuBrow S, Eberts EA, Murty VP. 2019. A common mechanism underlying choice's influence on preference and memory. Psychon Bull Rev 26: 1958-1966. doi:10.3758/s13423-019-01650-5

Duncan KD, Shohamy D. 2016. Memory states influence value-based decisions. J Exp Psychol Gen 145: 1420-1426. doi:10.1037/xge0000231

Duncan K, Semmler A, Shohamy D. 2019. Modulating the use of multiple memory systems in value-based decisions with contextual novelty. $J$ Cogn Neurosci 31: 1455-1467. doi:10.1162/jocn_a_01447

Fellows LK. 2017. The neuroscience of human decision-making through the lens of learning and memory. Curr Top Behav Neurosci 37: 231-251. doi:10.1007/7854_2016_468

Fenske MJ, Raymond JE. 2006. Affective influences of selective attention. Curr Dir Psychol Sci 15: 312-316. doi:10.1111/j.1467-8721 .2006.00459.x

Gluth S, Sommer T, Rieskamp J, Buchel C. 2015. Effective connectivity between hippocampus and ventromedial prefrontal cortex controls preferential choices from memory. Neuron 86: 1078-1090. doi:10.1016/j.neuron.2015.04.023

Gold JI, Shadlen MN. 2007. The neural basis of decision making. Annu Rev Neurosci 30: 535-574. doi:10.1146/annurev.neuro.29.051605.113038

Johnson EJ, Häubl G, Keinan A. 2007. Aspects of endowment: a query theory of value construction. J Exp Psychol Learn Mem Cogn 33: 461-474. doi:10.1037/0278-7393.33.3.461

Krajbich I, Armel C, Rangel A. 2010. Visual fixations and the computation and comparison of value in simple choice. Nat Neurosci 13: 1292-1298. doi:10.1038/nn.2635

Kuznetsova A. 2017. ImerTest package: tests in linear mixed effects models. $J$ Stat Softw 82. doi:10.18637/jss.v082.i13

Luettgau L, Tempelmann C, Kaiser LF, Jocham G. 2020. Decisions bias future choices by modifying hippocampal associative memories. Nat Commun 11: 3318. doi:10.1038/s41467-020-17192-7
Matuschek H, Kliegl R, Vasishth S, Baayen H, Bates D. 2017. Balancing type I error and power in linear mixed models. J Mem Lang 94: 305-315. doi:10 .1016/j.jml.2017.01.001

McGuire JT, Kable JW. 2014. Go means green. Nat Neurosci 17: 489-490. doi: $10.1038 / \mathrm{nn} .3680$

Mechera-Ostrovsky T, Gluth S. 2018. Memory beliefs drive the memory bias on value-based decisions. Sci Rep 8: 10592. doi:10.1038/ s41598-018-28728-9

Milosavljevic M, Malmaud J, Huth A, Koch C, Rangel A. 2010. The Drift Diffusion Model can account for the accuracy and reaction time of value-based choices under high and low time pressure. Judgm Decis Making 5: 437-449. doi:10.2139/ssrn.1901533

Murty VP, DuBrow S, Davachi L. 2015. The simple act of choosing influences declarative memory. J Neurosci 35: 6255-6264. doi:10.1523/JNEUROSCI $.4181-14.2015$

Salomon T, Botvinik-Nezer R, Gutentag T, Gera R, Iwanir R, Tamir M, Schonberg T. 2018. The cue-approach task as a general mechanism for long-term non-reinforced behavioral change. Sci Rep 8: 3614. doi:10 $.1038 / \mathrm{s} 41598-018-21774-3$

Salomon T, Botvinik-Nezer R, Oren S, Schonberg T. 2019. Enhanced striatal and prefrontal activity is associated with individual differences in nonreinforced preference change for faces. Hum Brain Mapp 41: 10431060. doi:10.1002/hbm.24859

Schonberg T, Katz LN. 2020. A neural pathway for nonreinforced preference change. Trends Cogn Sci 24: 504-514. doi:10.1016/j.tics.2020.04.002

Schonberg T, Bakkour A, Hover AM, Mumford JA, Nagar L, Perez J, Poldrack RA. 2014. Changing value through cued approach: an automatic mechanism of behavior change. Nat Neurosci 17: 625-630. doi:10.1038/nn.3673

Shadlen MN, Kiani R. 2013. Decision making as a window on cognition. Neuron 80: 791-806. doi:10.1016/j.neuron.2013.10.047

Shadlen MN, Shohamy D. 2016. Decision making and sequential sampling from memory. Neuron 90: 927-939. doi:10.1016/j.neuron.2016.04.036

Sharot T, Fleming SM, Yu X, Koster R, Dolan RJ. 2012. Is choice-induced preference change long lasting? Psychol Sci 23: 1123-1129. doi:10.1177/ 0956797612438733

Veling H, Chen Z, Tombrock MC, Verpaalen IAM, Schmitz LI, Dijksterhuis A, Holland RW. 2017. Training impulsive choices for healthy and sustainable food. J Exp Psychol Appl 23: 204-215. doi:10 .1037/xap0000112

Weber EU, Johnson EJ. 2006. Constructing preferences from memory. SSRN Electron J 1-26. doi:10.1017/CBO9780511618031.022

Weilbächer RA, Gluth S. 2017. The interplay of hippocampus and ventromedial prefrontal cortex in memory-based decision making. Brain Sci 7: 4. doi:10.3390/brainsci7010004

Yebra M, Galarza-Vallejo A, Soto-Leon V, Gonzalez-Rosa JJ, de Berker AO, Bestmann S, Oliviero A, Kroes MCW, Strange BA. 2019. Action boosts episodic memory encoding in humans via engagement of a noradrenergic system. Nat Commun 10: 3534. doi:10.1038/ s41467-019-11358-8

Zajonc RB. 1968. Attitudinal effects of mere exposure. J Pers Soc Psychol 9: 127. doi: $10.1037 / \mathrm{h} 0025848$

Zoltak MJ, Veling H, Chen Z, Holland RW. 2017. Attention! Can choices for low value food over high value food be trained? Appetite 124: 124-132. doi:10.1016/j.appet.2017.06.010

Received March 17, 2021; accepted in revised form July 14, 2021. 


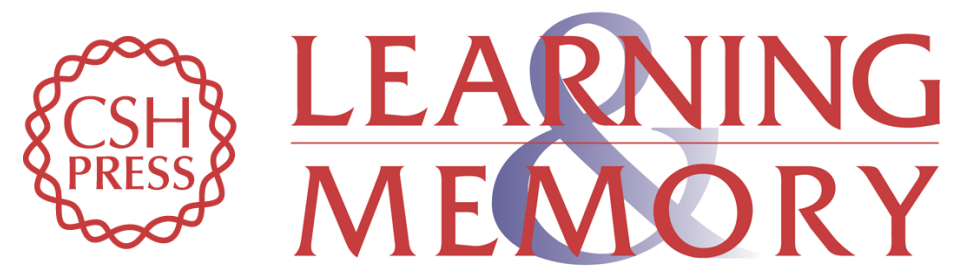

\section{Memory for individual items is related to nonreinforced preference change}

Rotem Botvinik-Nezer, Akram Bakkour, Tom Salomon, et al.

Learn. Mem. 2021, 28:

Access the most recent version at doi:10.1101//m.053411.121

\section{Supplemental http://learnmem.cshlp.org/content/suppl/2021/09/14/28.10.348.DC1 Material}

References This article cites 47 articles, 2 of which can be accessed free at: http://learnmem.cshlp.org/content/28/10/348.full.html\#ref-list-1

Creative This article, published in Learning \& Memory, is available under a Creative Commons Commons License (Attribution-NonCommercial 4.0 International), as described at License http://creativecommons.org/licenses/by-nc/4.0/.

Email Alerting Receive free email alerts when new articles cite this article - sign up in the box at the Service top right corner of the article or click here. 